\title{
Vorhofflimmern: Schlaganfallrisiko bei älteren Frauen besonders hoch
}

\author{
Etwa 1\% der Bevölkerung leidet \\ unter Vorhofflimmern. Die Häufigkeit \\ nimmt mit dem Alter dramatisch zu. \\ Patienten mit Vorhofflimmern haben \\ ein fünffach erhöhtes Schlaganfall- \\ risiko. Bisher durchgeführte epide- \\ miologische Studien zeigten ent- \\ weder ein höheres Risiko für Frauen \\ oder ein identisches Geschlechts- \\ verhältnis, daher war es notwendig, \\ weitere epidemiologische Daten für \\ diese Fragestellung zu gewinnen.
}

- Es handelt sich um eine populationsbezogene Kohortenstudie von Patienten im Alter von 65 Jahren und älter, die zwischen 1998 und 2007 wegen Vorhofflimmern ins Krankenhaus eingewiesen wurden. Die Erhebung stützt sich auf die Datenbanken der Krankenhäuser, der nachbehandelnden Ärzte und der in der Folgezeit ausgestellten Rezepte. Der primäre Endpunkt war das Schlaganfallrisiko.

Die Kohorte bestand aus 39398 Männern und 44115 Frauen. Zum Zeitpunkt der Aufnahme in das Krankenhaus waren Frauen älter und hatten einen höheren CHADS2-Score (1,99 vs. 1,13). 30

Akute StrahlenProktitis mit petechialen Blutungen und geröteter Rektum-Schleimhaut.
Tage nach der Entlassung bekamen $58,2 \%$ der Männer und 60,6\% der Frauen eine orale Antikoagulation mit Warfarin. Die Adhärenz an die Behandlung mit Warfarin war in beiden Behandlungsgruppen gut. Das Risiko eines Schlaganfalls betrug bei Frauen 2,02 pro 100 Patientenjahre, bei Männern 1,61 pro 100 Patientenjahre. Dieser Unterschied war statistisch signifikant. In einer multivariablen Regressionsanalyse hatten Frauen ein um 14\% höheres Schlaganfallrisiko als Männer, auch wenn dies für andere Risikofaktoren und vaskuläre Erkrankungen korrigiert wurde.

\section{- M. Avgil Tsadok et al.}

Sex differences in stroke risk among older patients with re-7cently diagnosed atrial fibrillation. JAMA 2012; 307: 1952-1958

\section{Kommentar}

Diese epidemiologische Studie belegt, was frühere Studien ebenfalls gezeigt hatten, dass nämlich ältere Frauen ein höheres Schlaganfallrisiko bei Vorhofflimmern haben als Männer. Daher ist es gerechtfertigt, weibliches Geschlecht in den Risiko-Score CHA2DS2-VASC2 aufzunehmen. Dies mag zum Teil daran liegen, dass Frauen im Alter über 75 Jahren signifikant seltener mit Vitamin-K-Antagonisten behandelt werden als Männer. Wenn sie mit Warfarin behandelt werden, ist die Adhärenz an die Behandlung deutlich besser als bei Männern. Mögliche Erklärungen für die Unterschiede könnten daran liegen, dass Frauen häufiger allein leben und dies dann gegebenenfalls die regelmäßige Kontrolle der INR-Werte erschwert.

H.-C. DIENER -

\section{Strahlen-Proktitis}

Bei einer externen Bestrahlung des Beckens entsteht durch die Schädigung der Mukosa und der Darmwand häufig eine so genannte Strahlen-Kolitis. Trotz der Fortschritte in der Strahlentherapie stellt diese unerwünschte
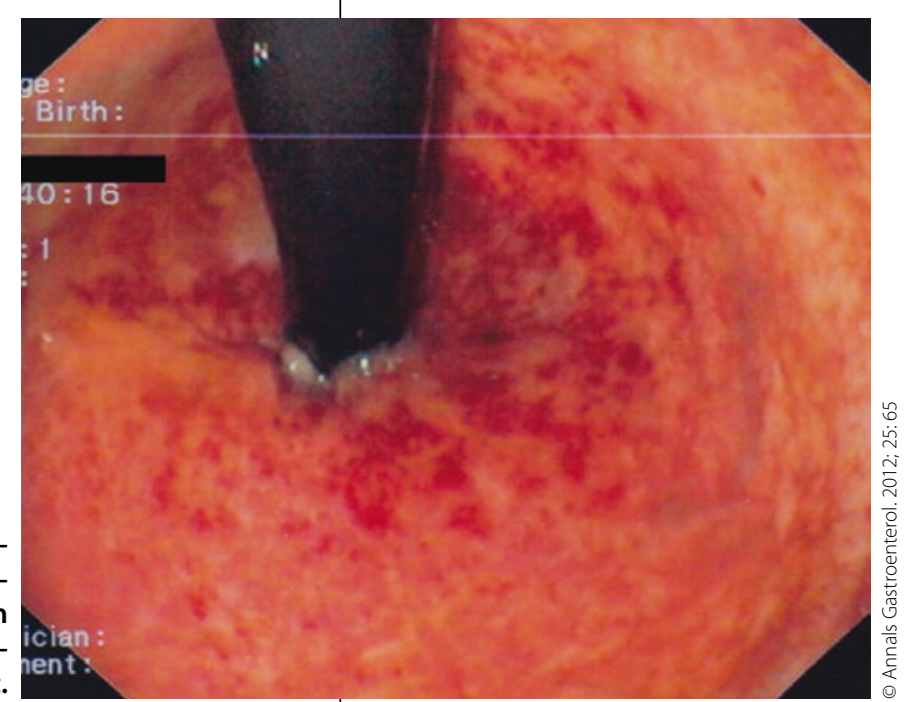

Nebenwirkung nach wie vor eine für den Patienten sehr belastende Begleiterscheinung vieler Beckenbestrahlungen dar. Behandlungsversuche sind Einläufe mit Steroiden, Mesalamin oder endoskopische Koagulationen. Im Fall des hier vorgestellten Patienten kam es drei Monate nach der Bestrahlung eines Prostata-Karzinoms zu blutigen Durchfällen. Endoskopisch wurde die Diagnose einer Strahlen-Proktitis gestellt (siehe Abb.) und der Patient mit gutem Erfolg mittels endoskopischer Koagulation und Mesalamin-Suppositorien behandelt. Nachdem Bestrahlungsfolgen am Kolon auch erst mehr als sechs Monate nach Beendigung der Bestrahlung auftreten können, gibt der Patient die Vorbehandlung bei der Anamnese möglicherweise nicht an, da er keinen Zusammenhang mehr sieht.

H. S. FÜESSL -

- K.Katsanos et al.

(Medical School of loannina, Korres.: etsianos@uoi.gr): Grading acute radiation bowel injury: an unadressed issue. Annals Gastroenterol. 2012; 25: 65 